International Journal of Engineering \& Technology, $7(2.23)(2018) 295-300$
International Journal of Engineering \& Technology
WPC
Website: www.sciencepubco.com/index.php/IJET
Research paper

\title{
Hilbert diagnostics of convective structures and phase transition in super cooled water
}

\author{
Yu. N. Dubnishchev 1, 2 *, V. A. Arbuzov 1, 2, E. V. Arbuzov ${ }^{2,3}$, V. S. Berdnikov 1, 2, \\ O. S. Melekhina ${ }^{2}$, V. V. Sotnikov ${ }^{1,2,4}$, A. A. Shibaev ${ }^{2}$ \\ ${ }^{1}$ Kutateladze Institute of Thermophysics Siberian Branch of the Russian Academy of Sciences, \\ Russia 630090, Novosibirsk, Pr. Ak. Lavrentyeva, \\ ${ }^{2}$ Novosibirsk State Technical University Russia 630073, Novosibirsk, Pr. K. Marksa, 20. \\ ${ }^{3}$ Sobolev Institute of Mathematics, Siberian Branch, Russian Academy of Sciences, \\ pr. Akademika Koptyuga 4, Novosibirsk, 630090 Russia \\ ${ }^{4}$ Technological Design Institute of Scientific Instrument Engineering, Siberian Division, \\ Russian Academy of Sciences, Novosibirsk, 630058 Russia \\ *Corresponding author E-mail: dubnistchev@itp.nsc.ru
}

\begin{abstract}
The evolution of the crystallization wave front and convective structures in a horizontal layer of supercooled water bounded by temperature-controlled flat surfaces is visualized using methods of Hilbert optics. The phase transition is manifested by the occurrence of a crystallization wave and is accompanied by a positive energy release, which, in turn, affects the dynamic distribution of the optical phase density gradient in supercooled water and induces phase perturbations in the probing light field. The results of measurements of the phase velocity and the shape of the crystallization front approximated by Bezier curves are presented. The wave front velocity is obtained using modified time-of-fight method. The phase velocity field is found to exhibit spatio-temporal quasi-periodicity that can be related to the existence of oscillatory phenomena in the crystallization process.
\end{abstract}

Keywords: Hilbert Optics; Optical Diagnostics of Flow; Convective Flows; Crystallization Wave.

\section{Introduction}

Optical diagnostic methods have had a significant impact on the development of modern experimental fluid dynamics see [1-5]. The application of optical diagnostics to convective flows is due to the particular importance of convection in geodynamics, atmos- pheric and oceanic physics, hydrodynamic and thermal processes with phase transitions, etc. Experimental studies of a plume gener- ated by a suddenly switched-on point [6] or linear source [7] have shown that the results of determination of the dynamics and shape of convective structures depend on the visualization method used. The velocity field of the visualized phase structure [8] in the liquid may differ significantly from the velocity field measured using particle image velocimetry [9] or laser Doppler velocimetry [10]. The interest in problems of crystallization waves in supercooled liquids and the evolution of convective structures in the water-ice system has considerably increased in recent years due to the inten- sification of the cyclic processes of ice melting and formation in the Arctic and Antarctic regions, which have a huge impact on the world's oceans and climate. In particular, the study of ice for- mation is of fundamental importance for the development of the Arctic shelf and gasbearing regions. Experimental and theoretical studies of the convective processes and dynamics of the ice water phase transition with consideration of the water density anomaly in the range of 0 $4^{\circ} \mathrm{C}$ are necessary for a wide range of technical and technological applications. Therefore, water can be used as a melt simulator for tuning single crystal growth techniques [11]. Results of convection studies for media with a density maximum can be found in [12].
The study of the dynamics of the phase tran- sition and the crystallization front is limited by the complexity of experimental designs and the need for adequate noninvasive measurement techniques. In this connection, optical diagnostic studies of the dynamics of the crystallization wave front in super cooled water at a solid boundary assume great importance [13]. The need for such studies has motivated the present work.

\section{Experimental methods}

Studying the dynamics of phase transitions in supercooled liquids and the evolution of convective structures in the water-ice system requires noninvasive diagnostic methods based on the latest advances in optics, laser techniques, and information technology. A sketch of the experimental setup comprising an optical diagnostic system and a test facility is shown in Fig. 1.

The illumination unit consists of a light source 1 (DRSh-250 mercury lamp), a condenser lens 2 , and a slit aperture 3 placed in the front focal plane of a lens 4 which produces a probing field in the test medium. This medium is water which fills a rectangular tank 5 with transparent glasses of optical quality. The internal dimensions of the tank are $136 \times 86 \times 30 \mathrm{~mm}$. The upper and lower walls are cooled plane-parallel copper plates of high quality $T_{1}$ and $T_{2}$.

The temperature of the upper plate is controlled and maintained by a thermostat in the range up to $-17^{\circ} \mathrm{C}$. The temperature of the lower plate is in the range from room temperature to $8,5^{\circ} \mathrm{C}$. The pipes feeding the coolant to the upper and lower plates are marked by numbers $8,9,10$, and 11 , and the drain pipes by numbers 12 and 13 . 
The feed pipes are symmetrically arranged relative to the drain pipes. The distance between the pipes is $80 \mathrm{~mm}$. The plates have internal cavities to which a cooling liquid is fed from thermostats 14 and 15.

(A)

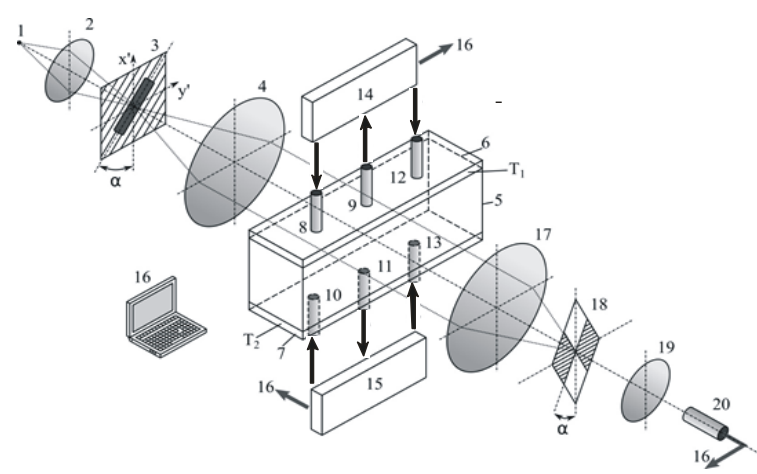

(B)

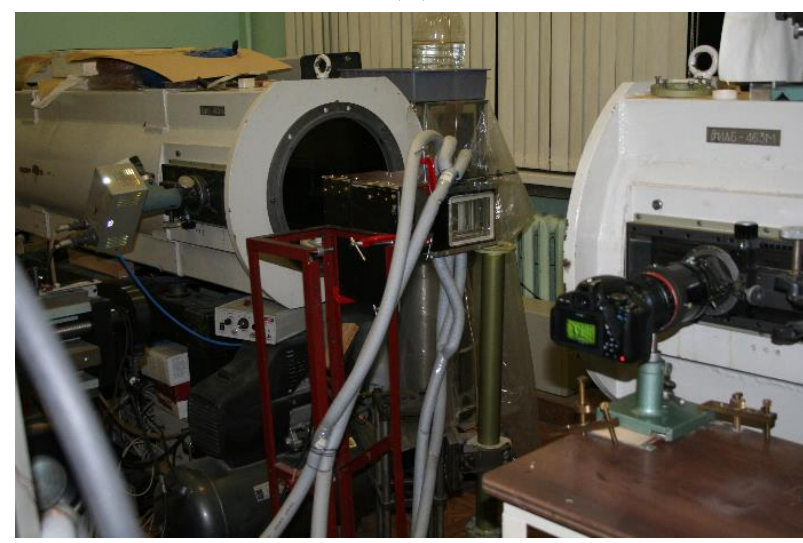

(C)
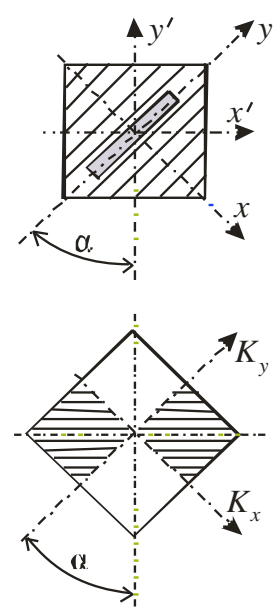

Fig. 1: Experimental setap: A diagram and $\mathbf{B}$ photo of the experimenta setup; relative position of the slit aperture and $\mathbf{C}$ the Hilbert filter.

The thermostats are controlled by a computer 16 in accordance with a predetermined program. Lens 17 generates a Fourier spectrum of the probing light field perturbed by the test medium in the frequency plane. A quadrant Hilbert filter 18 is placed in the Fourier plane of the lens 17. The frequency axis of the Hilbert filter is orthogonal to the direction of the image of the slit aperture of the light source $\left(\alpha=45^{\circ}\right)$. Lens 19 performs an inverse Fourier transform of the spatially filtered spectrum of the phase structure of the light field. The Hilbert image of the phase structure of the light field perturbed by the test medium reconstructed by the lens 19 is recorded by a camcorder connected to the computer. The optical diagnostic system is based on a commercial IAB-463M shadowgraph [8] comprising a light source unit, an optical spatial frequency filtering unit, and a unit for recording images of the visualized phase perturbations of the light field induced by the test medium; the units are modified according to the objectives of the experiments.

The probing field generated by the lens 4 from the slit light source passes through the test medium (water) in which the temperature boundary conditions $T_{1}$ and $T_{2}, T_{1}<T_{2}$, are set by the upper Plate 6 and lower Plate 7 temperature-controlled surfaces in accordance with a given program. Convective structures in the space between the upper and lower temperature-controlled surfaces appear as perturbations of the optical phase density fields. These structures are induced by Rayleigh-Benard convection complicated by the phase transition and the water density anomaly in the temperature range of $0 \div 4^{\circ} \mathrm{C}$. With increasing temperature gradient, water near the upper temperature-controlled surface becomes super cooled liquid, passing into a state of unstable equilibrium. In this medium, there is a transition from the liquid phase to the solid crystal state. This is a first-order phase transition. It manifests itself in the formation of a crystallization wave and is accompanied by energy release. This, in turn, affects the dynamic distribution of the optical phase density gradient in supercooled water and induces phase perturbations in the probing light field, whose Fourier spectrum $s\left(K_{x}, K_{y}\right)$ is formed in the frequency plane of the lens 17 . Here $K_{x}$ and $K_{y}$ are the spatial frequencies. Fig. 1c shows the orientation of the slit source aperture 3 and the orientation of the Hilbert filter 18. The $K_{y}$ axis is parallel and the $K_{y}$ axis is orthogonal to the direction of the slit aperture of the light source. The coordinate system $(x, y)$ is rotated about the coordinate system $\left(x^{\prime}, y^{\prime}\right)$ by an angle $\alpha=45^{\circ}$.

The coherent transfer function of the spatial frequency filter 18 performing a one-dimensional Hilbert transform is described by the expression

$H\left(K_{x}, K_{y}\right)=\left[e^{-i \varphi} \sigma\left(K_{x}\right)+e^{i \varphi} \sigma\left(-K_{x}\right)\right] \sigma\left(K_{y}\right)+$

$+\left[e^{i \varphi} \sigma\left(K_{x}\right)+e^{-i \varphi} \sigma\left(-K_{x}\right)\right] \sigma\left(-K_{y}\right)$,

where $\sigma\left( \pm K_{x}\right)$ and $\sigma\left( \pm K_{y}\right)$ are Heaviside functions; $\varphi$ is the phase shift defined by the corresponding quadrant of the spatial frequency filter. Given that,

$\sigma\left( \pm K_{x}\right)=\frac{1}{2}\left[1 \pm \operatorname{sgn} K_{x}\right]$,

where sgn $K_{x}$ is the sign function, expression Eq. (1) for the coherent transfer function of the filter takes the form

$\sigma\left(K_{x}\right)=\frac{1}{2}\left[1+\operatorname{sgn} K_{x}\right]$,

$H\left(K_{x}, K_{y}\right)=\cos \varphi-i\left[\sigma\left(K_{y}\right)-\sigma\left(-K_{y}\right)\right] \times$

$\times \sin \varphi \operatorname{sgn} K_{x}=\cos \varphi \operatorname{sgn} K_{x}$.

The filter with the coherent transfer function Eq. (2) performs a onedimensional Foucault-Hilbert transform. In the Fourier plane, $H\left(K_{x}, K_{y}\right)$, the spatial frequency axis $K_{x}$ is orthogonal to the image of the slit source. The Fourier spectrum of the light field immediately after the filter has the form

$s\left(K_{x}, K_{y}\right) H\left(K_{x}, K_{y}\right)=s\left(K_{x}, K_{y}\right) \times$

$\times \cos \varphi+\hat{s}_{x}\left(K_{x}, K_{y}\right)$.

Here $\hat{s}_{x}\left(K_{x}, K_{y}\right)=-i \operatorname{sgn}\left(K_{x}\right) s\left(K_{x}, K_{y}\right)$ is the Fourier spectrum of the light field subjected to the one-dimensional Hilbert transform on the $K_{x}$ axis. The phase shift $\varphi$ is a function of the wavelength $\lambda$ of the probing light field: $\varphi=\varphi(\lambda)$. At a wavelength $\lambda=\lambda_{0}$ that satisfies the condition, $\varphi\left(\lambda_{0}=\pi / 2\right)$, the coherent transfer function Eq. (2) takes the form

$H\left(K_{x}, K_{y}\right)=-i \operatorname{sgn} K_{x}$.

In this case, the filter performs a one-dimensional Hilbert transform:

$\hat{s}_{x}\left(K_{x}, K_{y}\right)=-i \operatorname{sgn} K_{x} s\left(K_{x}, K_{y}\right)$. 
If the investigated medium induces only phase perturbations of the probing field, the Fourier spectrum of the perturbed field is given by

$s\left(K_{x}, K_{y}\right)=e^{i \psi\left(K_{x}, K_{y}\right)}$.

Accordingly, for the Fourier spectrum of the filtered field, we have

$\hat{s}\left(K_{x}, K_{y}\right)=e^{i \psi\left(K_{x}, K_{y}\right)} H\left(K_{x}, K_{y}\right)$.

As follows from Eq. (6), the Fourier spectrum of phase perturbations has a structure consisting of isophase lines satisfying the equation, $\psi\left(K_{x}, K_{y}\right)=2 \pi m$, where $m=1,2,3 \ldots$.

Then $\widetilde{\Psi}\left(K_{x}, K_{y}\right)$ takes the meaning of a perturbation of the isophase line that broadens it:

$\widetilde{\psi}\left(K_{x}, K_{y}\right), \psi=2 \pi m+\widetilde{\Psi}\left(K_{x}, K_{y}\right), \widetilde{\psi} \ll 1$.

In this case, for the Fourier spectrum of the light field perturbed by the test medium, we have

$s\left(K_{x}, K_{y}\right)=\sum_{m} e^{i\left[\pi m+\widetilde{\psi}\left(K_{x}, K_{y}\right)\right]} \approx \sum_{m} e^{i \widetilde{\psi}_{m}\left(K_{x}, K_{y}\right)}$

or, taking into account that $\widetilde{\Psi}_{m}\left(K_{x}, K_{y}\right) \ll 1$,

$s\left(K_{x}, K_{y}\right)=\sum_{m}(-1)^{m}\left[1+i \widetilde{\Psi}_{m}\left(K_{x}, K_{y}\right)\right]$.

In view of Eq. (6), the filtered Fourier spectrum of the phase perturbations is expressed as

$s\left(K_{x}, K_{y}\right) H\left(K_{x}, K_{y}\right)=\left\{\sum_{m}(-1)^{m}\left[1+i \widetilde{\Psi}_{m}\left(K_{x}, K_{y}\right)\right]\right\} \times$

$\times\left[-i \operatorname{sgn} K_{x}\right]=\sum_{m} \widehat{\widetilde{\Psi}}_{m_{x}}\left(K_{x}, K_{y}\right)$,

where $\widehat{\widetilde{\Psi}}_{m_{x}}\left(K_{x}, K_{y}\right)$ is the Fourier spectrum of the phase perturbations visualized by means of the Hilbert transform on the $x$ axis. As follows from Eq. (8), phase structures are visualized by Hilbert filtering. Here it is taken into account that the Hilbert image of constant quantities is zero, according to the properties of the Hilbert transform.

The camcorder lens 19 performs an inverse Fourier transform of the filtered Fourier spectrum of phase perturbations:

$\sum_{m} \widehat{\widetilde{\Psi}}_{m_{x}}\left(K_{x}, K_{y}\right) \leftrightarrow \sum_{m} \widehat{\widetilde{\Psi}}_{m_{x}}(x, y)$.

The phase structures Eq. (9) visualized by the one-dimensional Hilbert transform operation are recorded by the camcorder CCD array:

$\left|\sum_{m} \widehat{\widetilde{\Psi}}_{m_{x}}(x, y)\right|^{2}=\sum_{m}\left|\widetilde{\widetilde{\psi}}_{m_{x}}(x, y)\right|^{2}$.

The one-dimensional Hilbert image of phase perturbations

$\sum_{m}\left|\widehat{\Psi}_{m_{x}}(x, y)\right|^{2}$

is analyzed in the coordinate system $\left(x^{\prime}, y^{\prime}\right)$ rotated about the coordinate system $\left(x^{\prime}, y\right)$ by an angle $\alpha=45^{\circ}$ :

$\sum_{m}\left|\widehat{\Psi}_{m_{x}}\left(x^{\prime}, y^{\prime}\right)\right|^{2}$

where

$x^{\prime}=\frac{1}{2}(x+y) ; y^{\prime}=\frac{1}{2}(x-y)$.
For $\varphi(\lambda) \neq \pi / 2 \varphi(\lambda)$, a one-dimensional Foucault-Hilbert transform with the coherent transfer function of the filter Eq. (2) is performed. The recorded image is a structure of isophase lines that represent the perturbations of the optical phase density in the test medium.

\section{Results}

With increasing temperature gradient, water near the upper temperature-controlled surface becomes supercooled liquid, passing into a state of unstable equilibrium.
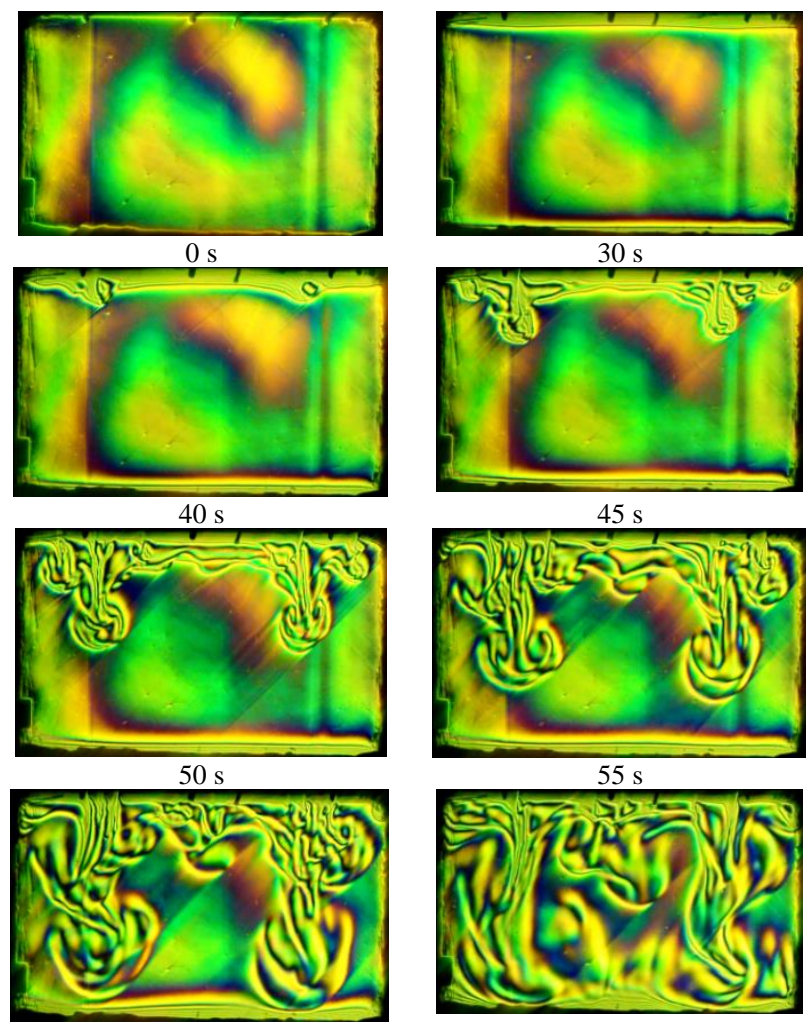

$60 \mathrm{~s}$

$70 \mathrm{~s}$

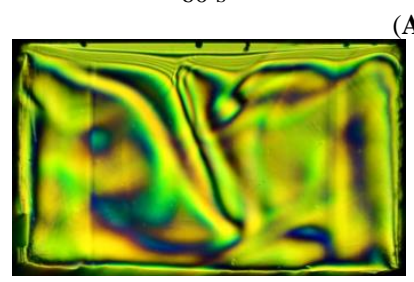

(A)

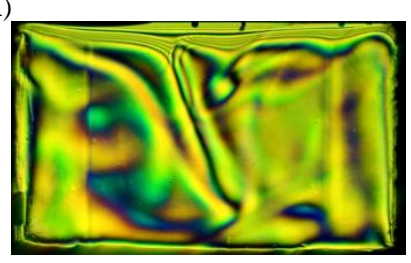

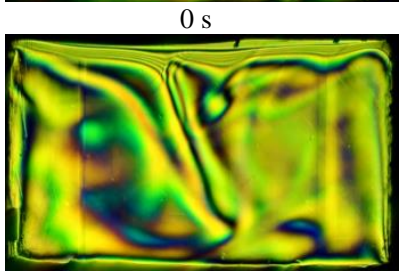

$0,8 \mathrm{~s}$

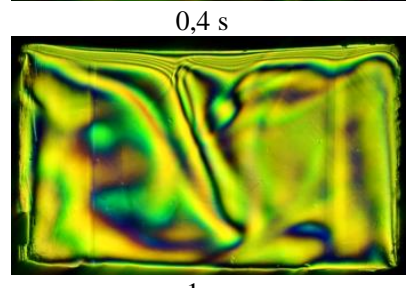

$1 \mathrm{~s}$
(B)

Fig. 2: Evolution of convective structures in the layer of the supercooled water: (A) the range of temperatures in the neighborhood of the density inversion $+11,1 \mathrm{C} \div+16,6^{\circ} \mathrm{C} ;(\mathbf{B})$ the phase transition liquid-solid body $15,1^{\circ} \mathrm{C} \div+8,9^{\circ} \mathrm{C}$.

This medium can undergo transition from the liquid phase to the solid crystal state. This phase transition is manifested by the occurrence of a crystallization wave and is accompanied by a positive energy release, which, in turn, affects the dynamic distribution of the optical phase density gradient in supercooled water and induces phase perturbations in the probing light field. As an example, Fig. 2a shows a video footage showing the evolution of convective structures in a water to the range of temperatures in the 
neighborhood of the density inversion. Example of convective structures in the layer of the supercooled liquid is shown in Fig. 2b and Fig. 3.

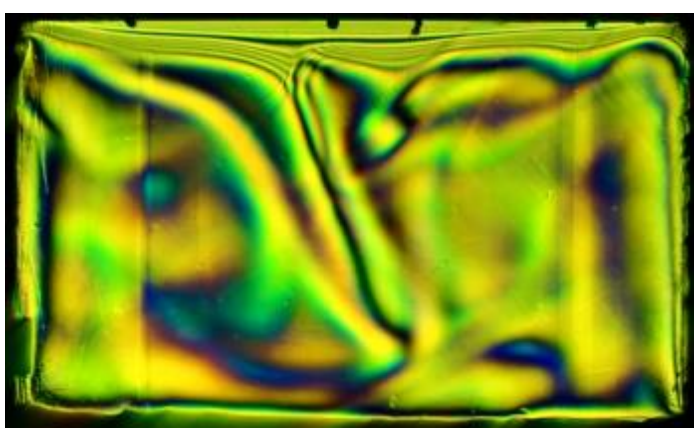

$0 \mathrm{~s}$

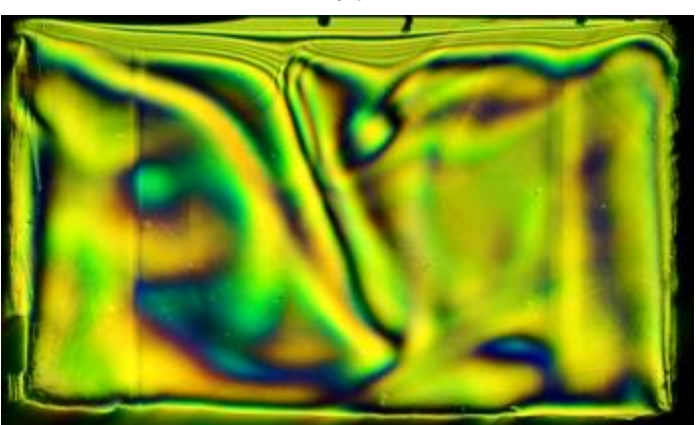

$0,4 \mathrm{~s}$

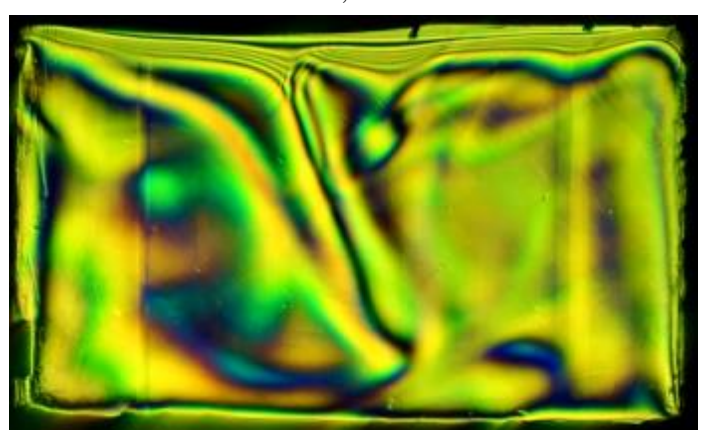

$0,8 \mathrm{~s}$

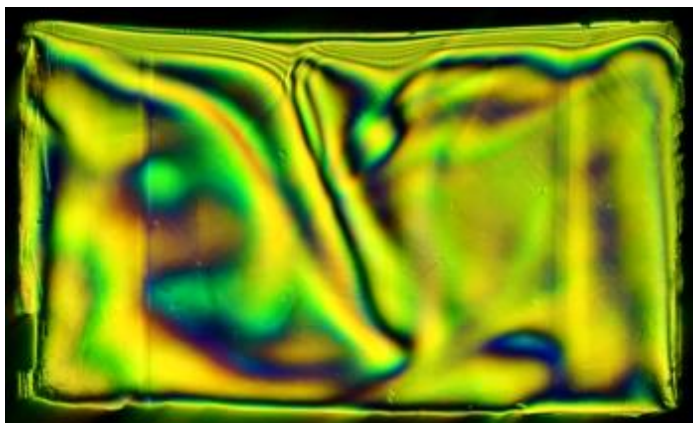

$1 \mathrm{~s}$

Fig. 3: The Phase Transition Liquid-Solid Body $-15,1^{\circ} \mathrm{C} \div+8,9^{\circ} \mathrm{C}$ (Fig. 2(B) on an Enlarged Scale).

Near, the top surface of the thermostat is clearly visible structure of the Hilbert bands displaying isotherms. The same figure clearly shows the crystallization wave front which represents the ice-water dynamic interface in the vicinity of the temperature-controlled flat plate bounding the supercooled water layer from above. Since the ice growth mechanism in highly supercooled water is largely determined by interfacial kinetics, it is of interest to estimate the dynamic shape of the crystallization wave front. For this, the crystallization wave front was approximated using Bernstein polynomials.

In the space between the upper and lower temperature-controlled surfaces, convective structures appear as Hilbert-visualized fields of the optical phase density.
They are induced by Rayleigh-Benard convection complicated by the phase transition and the water density anomaly in the temperature range of $0-4^{\circ} \mathrm{C}$. The lines of the crystallization wave front were approximated using Bernstein polynomials [8], as in the case of isotherms. The dynamics of the phase transition was determined at control points which divided each realization of the wave front approximated by a Bezier curve into parts with a predetermined ratio. The first control point was at the interface between the water and the temperature-controlled surface. The evolution of the crystallization wave front was reconstructed by comparing the coordinates of the control points in a video sequence taking into account the inter-frame time interval.

(A)
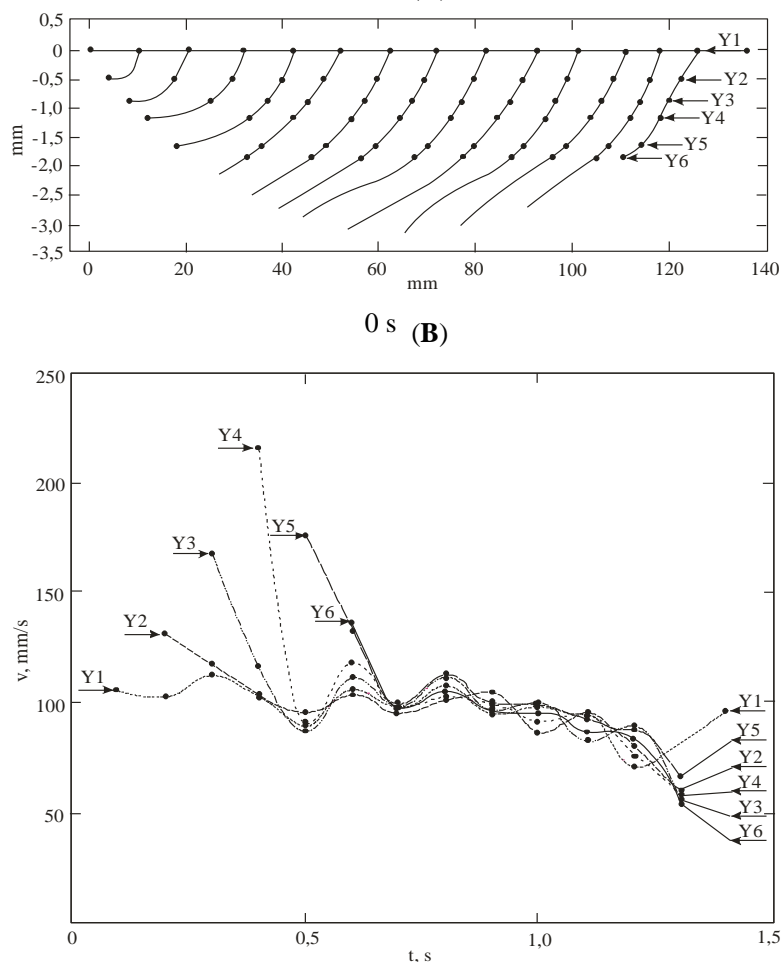

$0,4 \mathrm{~s}^{(\mathbf{C})}$

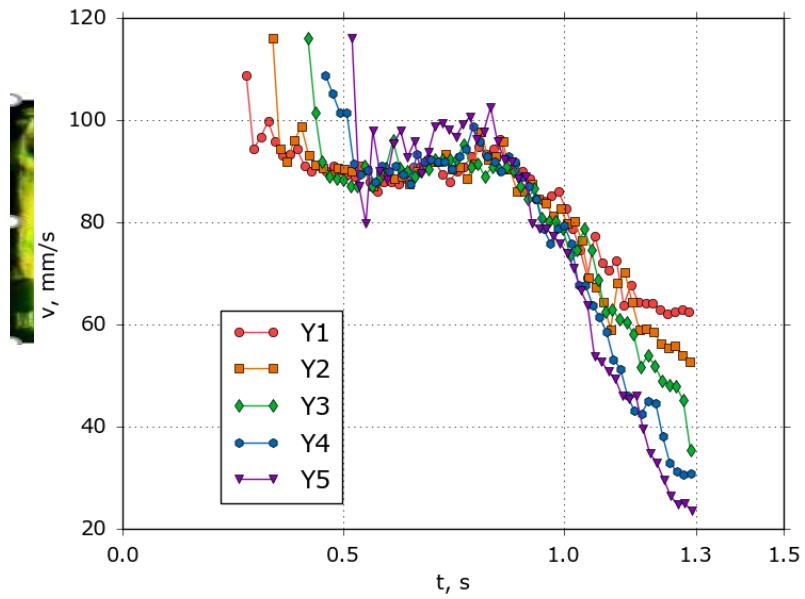

Fig. 4: Evolution of the crystallization wave front: (A) wave fronts; (B) and $(\mathbf{C})$ horizontal component of the phase velocity at the control points of the wave front.

Evolution of crystallization wave fronts on a modified (for clarity) scale along the vertical axis is shown in Fig. 4. It is clearly seen how the shape of the crystallization wave front changes during wave propagation. Figure 4a shows the reconstruction of the crystallization fronts using Bezier curves with the control points defined by the intersection of these fronts fixed at $0,1 \mathrm{~s}$ intervals with planes parallel to the temperature-controlled surface. These control points are generated so that each of the parallel planes passes through the 
beginning of the corresponding crystallization wave front approximated by a Bezier curve. The first plane coincides with the temperature-controlled surface. Figure $4 \mathrm{~b}$ illustrates the evolution of the phase velocity at the control points of the wave front that are selected according to Fig. 4a. Fig. 4c shows velocity simple moving averages with window size of 15 and averaging time interval of 0,3 s. The measurements of wave front velocity at the control points were carried out using modified time-of-flight method. Image processing with [14] edge detection operator was conducted with a view to simplification of the further analysis. Then the obtained time-series images were examined using defined pixel rows. The velocity estimation error on time interval related to fps of the camcorder is determined by pixel position error at the defined level. Five pixel rows with 2 pixel gaps between them were selected in order to measure wave front velocity. Simple moving averages with window size of 15 and averaging time interval of $0,3 \mathrm{~s}$ were computed to identify velocity trends.

Assessment of the relative velocity measurement error is $10 \%$. The quasiperiodic nature of the phase velocity curves at the contro points of the wave front indicates the existence of oscillatory phenomena in the crystallization process.

\section{Conclusion}

Methods of Hilbert optics were used to visualize convective structure and study the dynamics of the phase transition in a horizontal layer of super cooled water bounded by temperature-controlled flat surfaces. Since the mechanism of ice growth in supercooled water is substantially determined by the interfacial kinematics, it is of interest to estimate the dynamic form of the crystallization wave front. For this, the visualized front of the crystallization wave was approximated by Bernstein polynomials and Bezier curves. Normals to these curves at given points determine velocity vectors. Use of Bernstein polynomials to approximate isolines allows the structure of fringe patterns, shadowgrams, schlieren and Hilbert images to be defined analytically using a small number of parameters. Parametric definition of isolines makes it possible to determine the coordinates of the centers of Hilbert ranges at any section of the analyzed image, construct a mesh adaptive to deformations of the structure under study, and, applying a twodimensional interpolation, to reconstruct the phase density gradient field. Velocity vectors are determined from the shift of isolines in time intervals corresponding to the interframe intervals in the video sequence displaying the dynamic state of the test medium. The dynamics of the phase transition was investigated by measuring the velocity of the crystallization front at control points. The control points were defined as the points of intersection of the crystallization wave front and planes parallel to the temperature- controlled surface. The evolution of the crystallization wave front approximated by Bezier curves was reconstructed by comparing the coordinates of control points in the video sequence with the interframe time interval taken into account The velocity components parallel to the temperature-controlled plane of the heat exchanger were measured at the control points. Thus, the dynamic form of the crystallization front was determined. Measurements of the phase velocity and the crystallization front approximated by Bezier curves were performed. The studies were carried out for asymmetric arrangements of the pipes providing coolant flow in and out of the cavities of the copper plates bounding the horizontal water layer. The dynamics of the crystallization front was first studied. It has been found that there is a spatio- temporal realization of the crystallization wave front that is a velocity isoline. The obtained results are confirmed by velocity measurements at the control points of the crystallization front made by dynamic spatial filtering with processing of the digitized video stream in the reference frame specified by the pixel structure of the CCD array. It has been shown that the time dependence of the phase velocity at the control points of the wave front has a quasi-periodic nature. The quasi-periodic nature of the velocity at the control points of the wave front apparently reflects the dynamics of the interface induced by the motion of the crystallization front under the given boundary conditions.

\section{Acknowledgement}

This work was supported by the Russian Foundation for Basic Research (RFBR), project No. 14-08-00818.

\section{References}

[1] Merzkirch W (1987) Flow visualization, 2nd edn. Academic, Orlando.

[2] Settles GS (2001) Schlieren and shadowgraph techniques.Springer, Heidelberg.

[3] Smits A, Lim T (2000) Flow Visualization. Techniques and Examples. Imperial College Press. London:306.

[4] Kleine H (2013) Schlieren imagin and the real world. J. Visualization16:193-199.

[5] Gebhart B, Bendell VS, Shaullatullah H (1979) Buoyancy-induced flows adjacent to horizontal surfaces in water near its density ex- tremum. Int. J Heat Mass Tran22:132-149.

[6] Lappa M. (2010) Thermal Convection: Patterns, Evolution and Stability. Wiley.

[7] Davaille A, Limare A, Touitou F, KumagaiI, Vatterville J. (2011) Anatomy of a Laminar Starting Thermal Plume at High Prandtl Number. Exp. Fluids50:285-300.

[8] Arbuzov V, Arbuzov E, Berdnikov V, Bufetov N, Dubnishchev Yu, Shlapakova E. (2014) Optical Diagnostics of the Structure and Evolution of Buoyant Jets in a High-Velocity Fluid. Optoelectron. Instrum. Data Process50(5):466-473.

[9] Raffel M, Willert CT, Wereley ST, Kompenhans Yu. (2007) Particle Image Velocimetry. A Practical Guide. Berlin: Springer448.

[10] Dubnishchev Yu, Chugui Yu, Kompenhans Yu (2009) Laser Doppler Visualization of the Velocity Field with Elimination of the Influence of Multipaticle Scattering. Quantum Elektronica. 39 (10):962-966.

[11] Berdnikov V, Prostomolotov A, Verezub N, and Vinokurov V. (2015) Heptadecane and Gallium Crystallization in Hydrodynamic Czochralski Model. Journal of Materials Science and Engineering A 5 (9-10)351-360.

[12] Roy S (1972) Free Convection in Liquids under Maximum Density Condition. Indian J Phys. 46(6):245-249.

[13] V. A. Arbuzov, E. V. Arbuzov, V. S. Berdnikov, Yu. N. Dubnishchev, O. S. Melekhina. Optical Hilbert Diagnostics of Convective Structures and Phase Transition in a Horizontal Layer of Supercooled Water. Technical Physics, 2017, 62, (10):1599-1601.

[14] Canny L (1986) A Computational Approach to Edge Detection. IEEE Trans. Pattern Analysis and Machine Intelligence. 8(6):679698. 